\title{
The Prognostic Values of Preoperative Tumor Volume and Tumor Diameter in T1NO Papillary Thyroid Cancer
}

\section{Seung Taek Lim, MD \\ Ye Won Jeon, PhD \\ Young Jin Suh, MD, PhD}

\begin{abstract}
Purpose
The current TNM staging system for papillary thyroid cancer (PTC), which is based on tumor diameter, may not precisely reflect the true tumor burden. Therefore, we investigated whether preoperative tumor volume might more accurately reflect tumor burden and predict prognosis in patients with T1NO PTC than preoperative tumor diameter.
\end{abstract}

\section{Materials and Methods}

We retrospectively reviewed data from 1,659 patients with T1NO PTC, and after exclusion, a total of 1,081 patients were ultimately included. Tumor volume (V) was calculated for all patients using preoperative ultrasonography, and patients were grouped according to tumor diameter (T1a vs. T1b) and tumor volume (V1a vs. V1b). The recurrence-free survival (RFS) rates were then compared for these groups.

\section{Results}

The mean follow-up time was $66.12 \pm 28.75$ months, and $97.2 \%$ of the cohort experienced RFS. The optimal volume cut-off was defined as $0.545 \mathrm{~cm}^{3}$. There were no differences in RFS rates between T1a/T1b groups (all ages) and V1a/V1b groups ( $<45$ years of age). However, $\geq 45$-year-old patients in the V1b group had a significantly poorer RFS rate than those in the V1a group. These results were confirmed by multivariate analysis.

\section{Conclusion}

Our results indicate that preoperative tumor volume may be more useful for predicting prognosis than tumor diameter in $\geq 45$-year-old patients with T1NO PTC.

Correspondence: Young Jin Suh, MD, PhD

Division of Breast and Thyroid Surgical

Oncology, Department of Surgery,

St. Vincent's Hospital, College of Medicine,

The Catholic University of Korea,

93 Jungbu-daero, Paldal-gu, Suwon 16247, Korea

Tel: 82-31-249-8169

Fax: 82-31-247-5347

E-mail: yjsuh@catholic.ac.kr

Received July 22, 2016

Accepted November 22, 2016

Published Online December 13, 2016

\section{Key words}

Papillary thyroid carcinoma, Volume, Diameter, Recurrence, Prognosis

\section{Introduction}

Papillary thyroid cancer (PTC) is the most common histological subtype of thyroid carcinoma, accounting for $>80 \%$ of all thyroid malignancies [1-4]. Improvements in diagnostic modalities (including ultrasonography) have led to a recent and rapid increase in the detection of PTC, especially nonsymptomatic, node-negative, early-stage PTC [5-8]. As defined by the current American Joint Committee on Cancer
(AJCC) TNM staging system, T1 PTCs have a diameter of $\leq 2 \mathrm{~cm}$ [9]. They are further subclassified as T1a $(\leq 1 \mathrm{~cm})$ or $\mathrm{T} 1 \mathrm{~b}(>1 \mathrm{~cm}$ but $\leq 2 \mathrm{~cm})$, which can influence a patient's treatment plan. Moreover, the 2015 revision of the American Thyroid Association guidelines permits active surveillance instead of immediate surgery in select patients with an intrathyroid tumor diameter of $\leq 1 \mathrm{~cm}$ [10].

Although tumor diameter is widely used as the standard for treatment planning, it may not precisely reflect the true tumor burden because tumors do not always have a spheri- 
cal shape. For example, tumors that are relatively long and narrow would have a lesser tumor volume and burden than those with a diameter equal to that of cylindrical tumors. Ultrasonography has shown that PTCs are typically long and narrow. Therefore, we investigated whether tumor volume would more accurately reflect tumor burden, resulting in more accurate prognosis, compared with tumor diameter. To accomplish this, prognostic values of preoperative tumor volumes were assessed and compared to those of the conventional staging system based on tumor diameter in patients with T1N0 PTC.

\section{Materials and Methods}

\section{Patients, follow-up, and measurements}

This study's retrospective design was approved by the Institutional Review Board of St. Vincent's Hospital. We retrospectively reviewed data from 2,585 patients who were diagnosed with thyroid cancer and treated at St. Vincent's Hospital between February 1982 and December 2012. All patients were treated by total thyroidectomy or lobectomy with isthmectomy, and central lymph node dissection was performed when lymph node metastasis was suspected during the preoperative evaluation. The postoperative pathological reports confirmed that all patients had tumor-free margins. Thyroid-stimulating hormone suppression therapy was administered during the follow-up period, and nonstimulated serum thyroglobulin measurements and neck ultrasonography (with or without neck and chest computed tomography) were routinely performed every 6-12 months.

Among the eligible patients, we identified 1,659 patients with T1N0 PTC. However, to create a homogenous study population, we excluded patients with aggressive histological variants (solid, insular, columnar, tall cell, or diffuse sclerosing), lymphovascular invasion, multifocal malignancies, extrathyroidal extension, or capsular invasion, as well as those who had received postoperative radioactive iodine therapy. We also excluded patients who had a cystic tumor portion or poorly defined margins to ensure that the tumor volume could be accurately calculated. Thus, 1,081 patients were selected for inclusion in this study.

All 1,081 patients had undergone preoperative ultrasonography to examine the thyroid tumor. Three experienced radiologists, each with more than 5 years of thyroid ultrasound experience, had evaluated the tumor's dimensions (using transverse and sagittal orthogonal scans) and cervical lymph node status. The recorded dimensions were transverse length $(\mathrm{T})$, longitudinal length (L), and depth (D), which allowed us to calculate the tumor's volume (V) using the ellipsoid formula $(\mathrm{V}=\mathrm{T}[\mathrm{cm}] \times \mathrm{L}[\mathrm{cm}] \times \mathrm{D}[\mathrm{cm}] \times \pi / 6)$.

\section{Statistical analysis}

The clinicopathological variables that we evaluated included age at diagnosis, sex, surgery type, histological subtype, and tumor location. All patients were categorized according to tumor diameter as T1a $(\leq 1 \mathrm{~cm})$ or T1b $(>1 \mathrm{~cm}$ but $\leq 2 \mathrm{~cm}$ ). We next performed receiver operating characteristic (ROC) curve analysis to estimate the optimal cut-off tumor volume for predicting recurrence. Based on the result of the ROC analysis, we also categorized the patients according to tumor volume as V1a or V1b. To validate the results of the ROC analysis, an internal validation was performed using the 1,000 bootstrap resampling method.

The primary outcome of interest in the study was recurrence-free survival (RFS). RFS was defined as the period between the date of the initial surgery and the first instance of contralateral lobe, locoregional (the operative bed, trachea, strap muscle, or cervical lymph nodes), or distant recurrence or the last follow-up. The presence of a contralateral lobe or

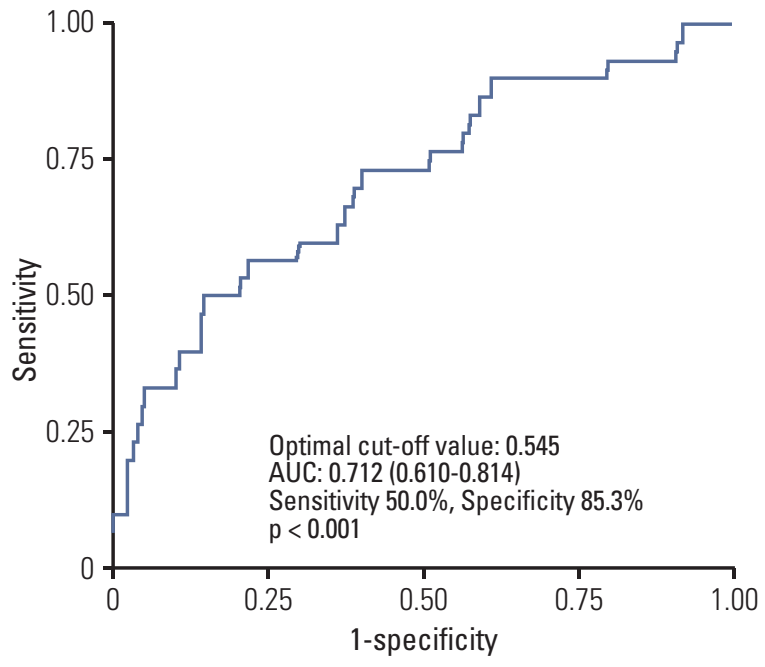

Internal validation-bootstrap

\begin{tabular}{lcc} 
Model & AUC & $95 \%$ Confidence interval \\
Orignal & 0.712 & $0.610-0.814$ \\
Bootstrap & 0.713 & $0.602-0.806$ \\
\hline
\end{tabular}

Statistics were carried out using logistic regression and calculated using bootstrap 1,000 replicates to estimate

Fig. 1. Receiver operating characteristic (ROC) curve analysis to estimate the optimal cut-off tumor volume for predicting recurrence. 
Table 1. Baseline patient characteristics

\begin{tabular}{|c|c|c|c|c|c|c|}
\hline \multirow{2}{*}{ Characteristic } & \multicolumn{3}{|c|}{ Tumor diameter } & \multicolumn{3}{|c|}{ Tumor volume } \\
\hline & T1a & $\mathrm{T} 1 \mathrm{~b}$ & p-value & V1a & V1b & p-value \\
\hline \multicolumn{7}{|l|}{ Tumor size $(\mathrm{cm})$} \\
\hline Mean \pm SD & $0.64 \pm 0.19$ & $1.38 \pm 0.27$ & & $0.69 \pm 0.24$ & $1.50 \pm 0.25$ & \\
\hline \multicolumn{7}{|l|}{ Sex } \\
\hline Female & $734(89.2)$ & $222(86.0)$ & 0.169 & $808(88.6)$ & $148(87.6)$ & 0.703 \\
\hline Male & $89(10.8)$ & $36(14.0)$ & & $104(11.4)$ & $21(12.4)$ & \\
\hline \multicolumn{7}{|l|}{ Age (yr) } \\
\hline Mean \pm SD & $48.00 \pm 10.50$ & $48.58 \pm 11.47$ & & $48.14 \pm 10.49$ & $48.15 \pm 12.03$ & \\
\hline$<45$ & $325(39.5)$ & $96(37.2)$ & 0.512 & $351(38.5)$ & $70(41.4)$ & 0.473 \\
\hline$\geq 45$ & $498(60.5)$ & $162(62.8)$ & & $561(61.5)$ & 99 (58.6) & \\
\hline \multicolumn{7}{|l|}{ Surgery } \\
\hline Lobectomy+isthmectomy & $590(71.7)$ & $115(44.6)$ & $<0.001$ & $635(69.6)$ & $70(41.4)$ & $<0.001$ \\
\hline Total thyroidectomy & $233(28.3)$ & $143(55.4)$ & & $277(30.4)$ & $99(58.6)$ & \\
\hline \multicolumn{7}{|l|}{ Histological subtype } \\
\hline Classic & $776(94.3)$ & $238(92.2)$ & 0.235 & $857(94.0)$ & $157(92.9)$ & 0.596 \\
\hline Follicular variant & $47(5.7)$ & $20(7.8)$ & & $55(6.0)$ & $12(7.1)$ & \\
\hline \multicolumn{7}{|l|}{ Location } \\
\hline Right & $439(53.3)$ & $139(53.9)$ & 0.062 & 485 (53.2) & $93(55.0)$ & 0.005 \\
\hline Left & $363(44.1)$ & $105(40.7)$ & & $404(44.3)$ & 64 (37.9) & \\
\hline Isthmus & $21(2.6)$ & $14(5.4)$ & & $23(2.5)$ & $12(7.1)$ & \\
\hline \multicolumn{7}{|l|}{ CLND } \\
\hline Performed & $466(56.6)$ & $147(57.0)$ & 0.920 & $514(56.4)$ & $99(58.6)$ & 0.593 \\
\hline Not performed & $357(43.4)$ & $111(43.0)$ & & 398 (43.6) & $70(41.4)$ & \\
\hline
\end{tabular}

Values are presented as number (\%) unless otherwise indicated. SD, standard deviation; CLND, central lymph node dissection.

locoregional recurrence was based on cytological or histopathological evidence of disease in specimens obtained by fine-needle aspiration biopsy or surgical excision. We did not use serum thyroglobulin levels to identify recurrent disease, as they do not definitively diagnose recurrent or persistent disease in patients who have not undergone total thyroidectomy.

Independent $t$ tests and chi-square tests were used to evaluate inter-group differences in the clinicopathological variables. Survival curves were created using the Kaplan-Meier method and compared using the log-rank test. Multivariate analyses were performed using Cox's proportional hazard regression model to assess the independent prognostic values of tumor volume and tumor diameter, and hazard ratios and $95 \%$ confidence intervals (CIs) were estimated for each variable. Because age is a known prognostic factor in PTC, we also conducted subgroup analyses using a cut-off age of 45 years. All statistical tests were two-sided, and p-values of $<0.05$ were considered to indicate significance. All statistical analyses were performed using the SPSS ver. 12.0 (SPSS Inc., Chicago, IL).

\section{Results}

\section{Cut-off tumor volume for predicting recurrence}

The ROC curve analysis (Fig. 1) revealed that the optimal cut-off volume for predicting tumor recurrence was 0.545 $\mathrm{cm}^{3}$, which provided an area under the curve of $0.712(95 \%$ CI, 0.610 to $0.814 ; \mathrm{p}<0.001$; sensitivity $50.0 \%$; specificity $85.3 \%)$. Therefore, patients were categorized as having a tumor volume of $\mathrm{V} 1 \mathrm{a}\left(\leq 0.545 \mathrm{~cm}^{3}\right)$ or V1b $\left(>0.545 \mathrm{~cm}^{3}\right)$. The cutoff value obtained from the ROC analysis was internally validated using the bootstrap method. The estimated concordance probability determined by 1,000 repetitions of the bootstrap sample corrections was 0.713 (95\% CI, 0.602 to 0.806).

\section{Comparison of patient and tumor characteristics among tumor groups}

The baseline characteristics of the study cohorts are shown in Table 1. The mean age of the entire cohort was $48.14 \pm 10.74$ 


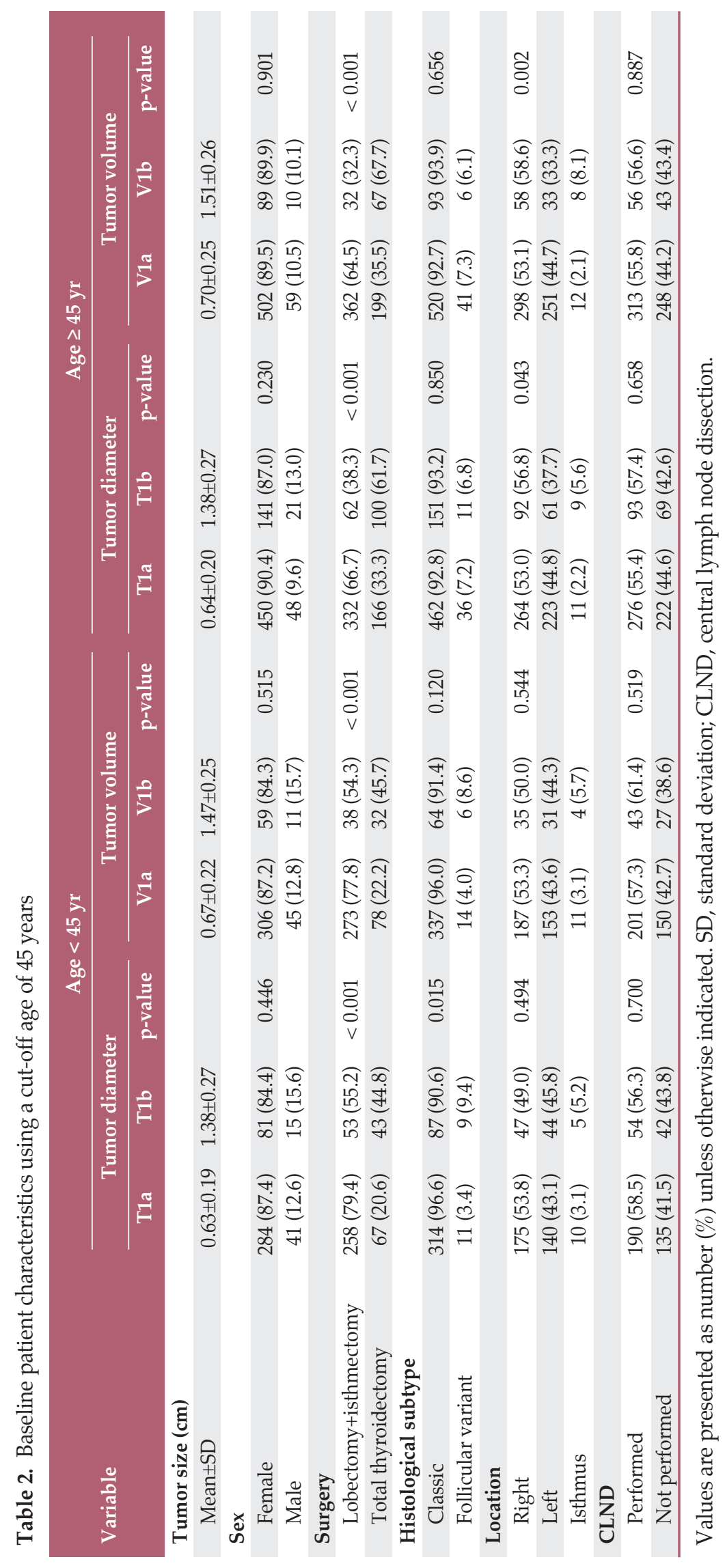

VOLUME 49 NUMBER 4 October 2017 
Table 3. Tumor recurrence patterns in the study population

\begin{tabular}{|c|c|c|c|c|c|c|c|c|}
\hline \multirow{3}{*}{ Variable } & \multicolumn{4}{|c|}{ Age $<45 \mathrm{yr}$} & \multicolumn{4}{|c|}{ Age $\geq 45 \mathrm{yr}$} \\
\hline & \multicolumn{2}{|c|}{ Tumor diameter } & \multicolumn{2}{|c|}{ Tumor volume } & \multicolumn{2}{|c|}{ Tumor diameter } & \multicolumn{2}{|c|}{ Tumor volume } \\
\hline & T1a & T1b & V1a & V1b & T1a & T1b & V1a & V1b \\
\hline \multicolumn{9}{|l|}{ Tumor recurrence } \\
\hline No & $319(98.2)$ & $92(95.8)$ & $345(98.3)$ & $66(94.3)$ & $489(98.2)$ & 151 (93.2) & $552(98.4)$ & $88(88.9)$ \\
\hline Yes & $6(1.8)$ & $4(4.2)$ & $6(1.7)$ & $4(5.7)$ & $9(1.8)$ & $11(6.8)$ & $9(1.6)$ & $11(11.1)$ \\
\hline \multicolumn{9}{|l|}{ Recurrence site } \\
\hline Contralateral lobe & $4(66.7)$ & $3(75.0)$ & $4(66.7)$ & $3(75.0)$ & $6(66.7)$ & $4(36.4)$ & $6(66.7)$ & $4(36.4)$ \\
\hline Locoregional & $2(33.3)$ & $1(25.0)$ & $2(33.3)$ & $1(25.0)$ & $2(22.2)$ & $7(63.6)$ & $2(22.2)$ & $7(63.6)$ \\
\hline Distant & 0 & 0 & 0 & 0 & $1(11.1)$ & 0 & 1 (11.1) & 0 \\
\hline
\end{tabular}

Values are presented as number $(\%)$.

years. Eighty-nine patients were moved from the T1b group to the V1a group ( 26 were aged $<45$ years, and 63 were aged $\geq 45$ years). Total thyroidectomy was more performed more often in the T1b group than in the T1a group (55.4\% vs. $28.3 \%$, respectively; $\mathrm{p}<0.001$ ). Moreover, comparison of groups V1b and V1a revealed significant differences in the number of right lobe tumors ( $55.0 \%$ vs. $53.2 \%$, respectively; $\mathrm{p}=0.005)$, isthmus tumors $(7.1 \%$ vs. $2.5 \%$, respectively; $\mathrm{p}=0.005)$, and total thyroidectomies ( $58.6 \%$ vs. $30.4 \%$, respectively; $\mathrm{p}<0.001$ ).

Age-based subgroup analyses (Table 2) showed that total thyroidectomy was significantly more frequent in $<45$-yearold patients in group T1b than group T1a (44.8\% vs. 20.6\%, respectively; $\mathrm{p}<0.001$ ) and group $\mathrm{V} 1 \mathrm{~b}$ than group V1a $(45.7 \%$ vs. $22.2 \%$, respectively; $\mathrm{p}<0.001)$. There were also significantly more histological variants in younger patients in group $\mathrm{T} 1 \mathrm{~b}$ than group $\mathrm{T} 1 \mathrm{a}(9.4 \%$ vs. $3.4 \%$, respectively; $\mathrm{p}=0.015$ ). When we compared the $\geq 45$-year-old patients in groups $\mathrm{T} 1 \mathrm{~b}$ and $\mathrm{T} 1 \mathrm{a}$, we found significant differences in the number of right lobe tumors (56.8\% vs. $53.0 \%$, respectively; $\mathrm{p}=0.043)$, isthmus tumors (5.6\% vs. $2.2 \%$, respectively; $\mathrm{p}=0.043)$, and total thyroidectomies $(61.7 \%$ vs. $33.3 \%$, respectively; $\mathrm{p}<0.001$ ). When we compared the $\geq 45$-year-old patients in groups V1b and V1a, we found similar differences in the number of right lobe tumors $(58.6 \%$ vs. $53.1 \%$, respectively; $\mathrm{p}=0.002$ ), isthmus tumors ( $8.1 \%$ vs. $2.1 \%$, respectively; $\mathrm{p}=0.002)$, and total thyroidectomies $(67.7 \%$ vs. $35.5 \%$, respectively; $\mathrm{p}<0.001$ ).

\section{Tumor recurrence patterns}

The mean follow-up time was $66.12 \pm 28.75$ months, and 97.2\% of the entire cohort experienced RFS. Table 3 provides detailed information regarding tumor recurrence in the study population. Thirty patients experienced tumor recur- rence, with 17 cases of contralateral lobe recurrence and 12 cases of locoregional recurrence. Only one patient experienced distant recurrence (lung metastasis), which was detected via chest computed tomography and confirmed by excisional biopsy. None of the patients who were moved from the $\mathrm{T} 1 \mathrm{~b}$ group to the V1a group experienced tumor recurrence.

\section{Analysis of the rates of RFS}

There was no significant difference in RFS rates between the T1a and T1b groups (98.2\% vs. $94.2 \%$, respectively; $\mathrm{p}=0.997$ ) or the V1a and V1b groups ( $98.4 \%$ vs. $91.1 \%$, respectively; $\mathrm{p}=0.253$ ) (Fig. 2A). Multivariate analysis provided similar findings (Table 4).

When we conducted subgroup analyses using a cut-off age of 45 years, no significant differences in RFS rates were observed between the $<45$-year-old patients in either the $\mathrm{T} 1 \mathrm{a} / \mathrm{T} 1 \mathrm{~b}$ groups $(98.2 \%$ vs. $95.8 \%$, respectively; $\mathrm{p}=0.137$ ) or the V1a/V1b groups ( $98.3 \%$ vs. $94.4 \%$, respectively; $\mathrm{p}=0.236$ ) (Fig. 2B and C). However, $\geq 45$-year-old patients in the V1b group showed significantly poorer RFS rates than those in the V1a group ( $88.9 \%$ vs. $98.4 \%$, respectively; $\mathrm{p}=0.029$ ) (Fig. 2C). Multivariate analyses revealed no significant agerelated differences in RFS rates between the T1a and $\mathrm{T} 1 \mathrm{~b}$ groups. However, poorer RFS rates were associated with older patients in group V1b than V1a upon multivariate analysis (hazard ratio, 2.676; 95\% CI, 1.075 to $6.661 ; \mathrm{p}=0.034$ ) (Table 4). 

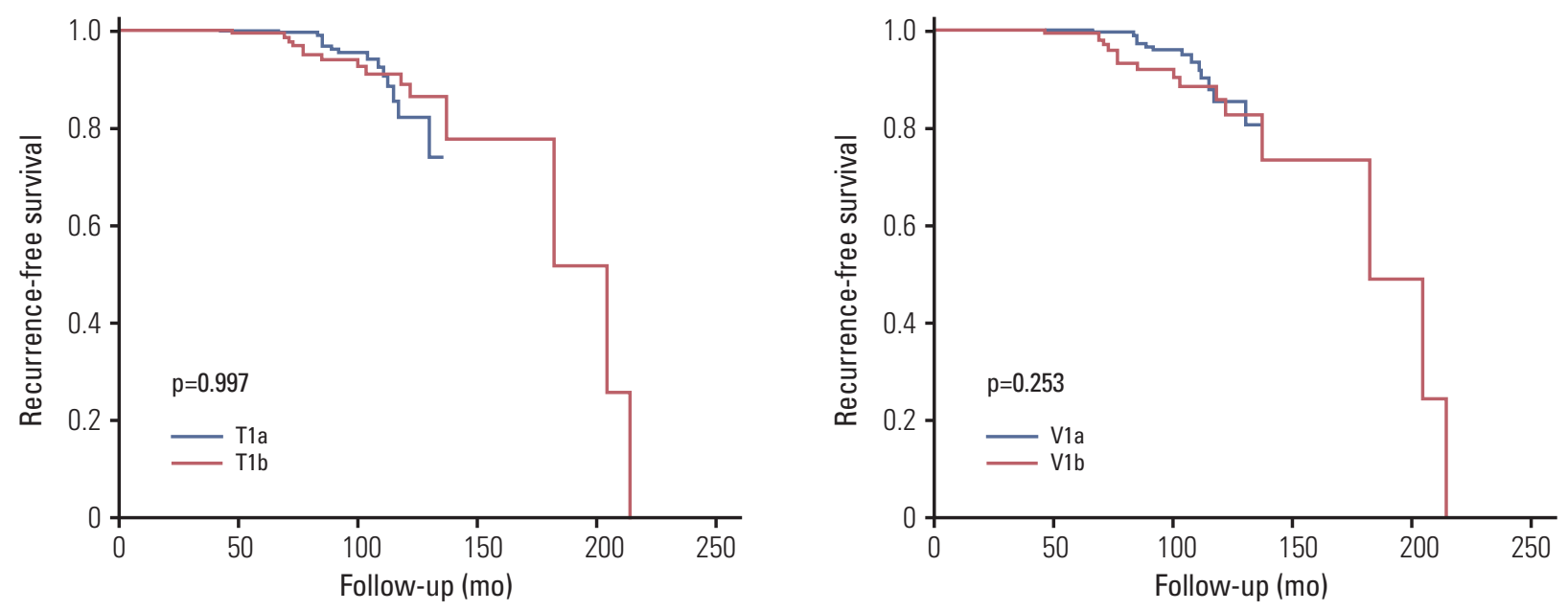

A
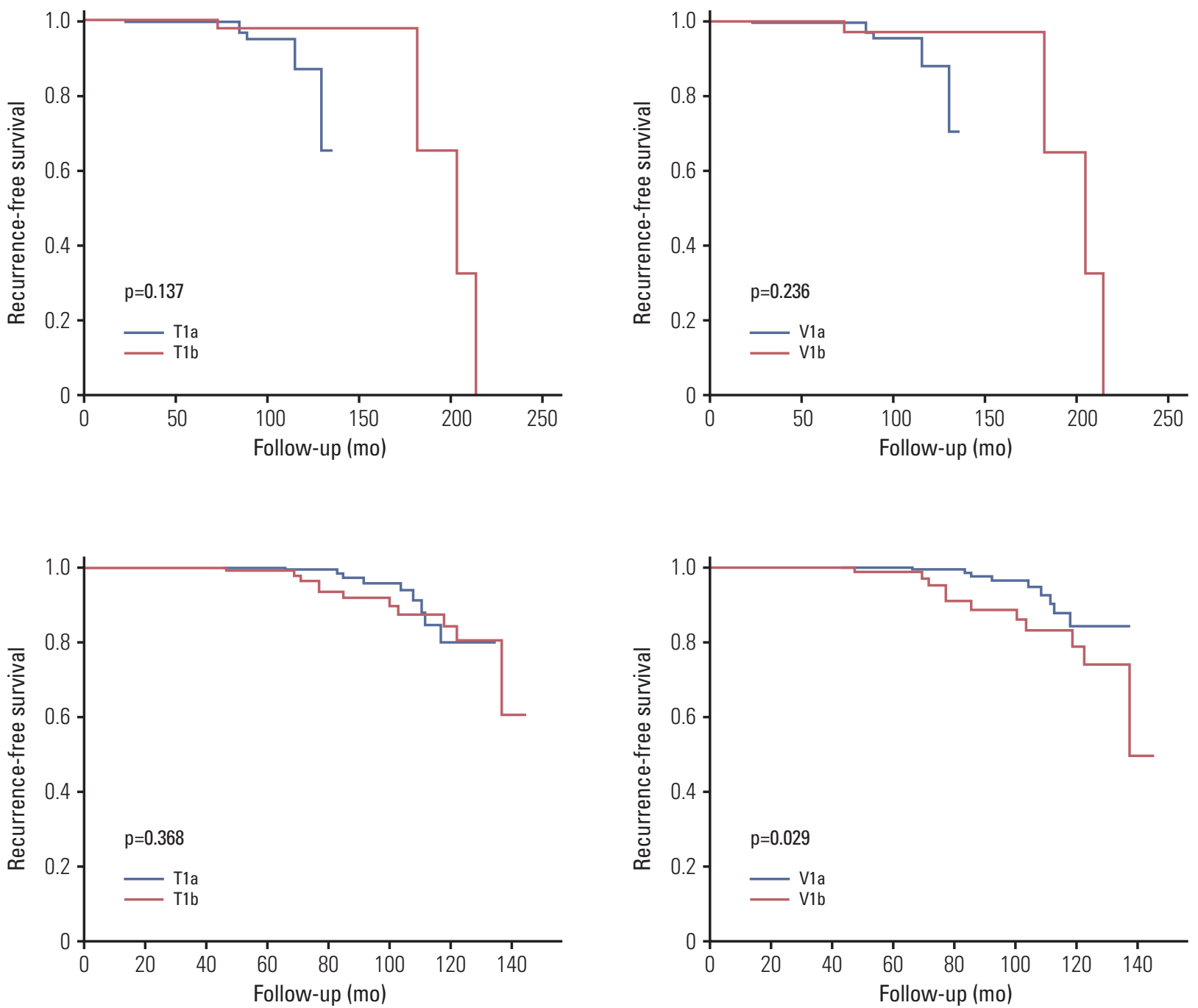

Fig. 2. Recurrence-free survival according to tumor diameter (T) and tumor volume categorization (V). Entire study population (A), patients aged $<45$ years $(B)$, and patients aged $\geq 45$ years $(C)$. 
Table 4. Multivariate analysis of tumor recurrence

\begin{tabular}{l} 
Variable \\
\cline { 2 - 3 }
\end{tabular}

$\mathrm{HR}$, hazard ratio; CI, confidence interval. ${ }^{\mathrm{a})}$ Adjusted for surgery, ${ }^{\mathrm{b})}$ Adjusted for surgery, histologic subtype, ${ }^{\mathrm{c})}$ Adjusted for surgery, tumor location.

\section{Discussion}

The present study assessed the prognostic value of preoperative tumor volume and compared it to that of the conventional staging system based on tumor diameter in patients with T1N0 PTC. We found that tumor volume better predicted tumor recurrence than the conventional staging system in $\geq 45$-year-old patients with T1N0 PTC. Accurately measuring the tumor's diameter may be hindered by the fact that tumors exhibit various shapes; hence, tumor volume may more precisely reflect tumor burden. Several studies have evaluated the prognostic value of tumor volume and tumor diameter in non-thyroid cancers, including non-small cell lung cancer and oropharyngeal cancer [11,12]. Furthermore, Park et al. [13] reported an association between tumor volume and occult central lymph node metastasis in PTC. However, to the best of our knowledge, this is the first study to compare the prognostic value of preoperative tumor diameter and tumor volume in early-stage node-negative papillary cancer.

There is debate regarding whether patients with T1a papillary cancer have a different prognosis than those with $\mathrm{T} 1 \mathrm{~b}$ papillary cancer [14-16]. Although the current AJCC staging system subdivides $\mathrm{T} 1$ disease into $\mathrm{T} 1 \mathrm{a}$ and $\mathrm{T} 1 \mathrm{~b}$ based on a tumor diameter of $1 \mathrm{~cm}$, this subdivision does not affect the final tumor stage as the seventh version of the AJCC staging system defines both $\mathrm{T} 1 \mathrm{a}$ and $\mathrm{T} 1 \mathrm{~b}$ disease as stage 1 for nodenegative patients who are aged $\geq 45$ years. This raises the question of whether it is necessary to subdivide T1 disease into T1a and T1b. However, we found a difference in prognosis when we compared $\geq 45$-year-old patients in the V1a and $\mathrm{V} 1 \mathrm{~b}$ groups. Therefore, it may be useful to consider subdividing stage 1 PTC according to tumor volume, especially in patients aged $\geq 45$ years, to more precisely predict their prognosis.
In the present study, 89 patients were reclassified from $\mathrm{T} 1 \mathrm{~b}$ to V1a based on their tumor volume, and none of these patients experienced tumor recurrence. These findings highlight the possibility of misclassification in the current staging system, which is based on tumor diameter. Our results suggest that use of tumor volume for staging might reduce the possibility of misclassification. In addition, it may be appropriate to include tumor volume as a criterion for active surveillance of low-risk PTC in the 2015 revision of the American Thyroid Association guidelines, which currently only consider tumor diameter.

It should be noted that this study has several limitations. First, its single-center retrospective design has well-known risks of bias. Second, discordance between tumor volumes measured via preoperative ultrasonography versus pathology is possible, as is interobserver variation during the approximately 30 -year data collection period. However, several studies have reported that preoperative ultrasonography reliably estimates the size of thyroid cancers and has low interobserver variability $[17,18]$. Third, the relatively short follow-up period and limited number of recurrences might limit the statistical power of our analysis. Therefore, larger studies with prolonged follow-ups should be conducted to validate our findings. Finally, we did not consider genetic data (e.g., BRAF mutation status), which can be useful for predicting prognosis in patients with PTC.

Our findings show that preoperative tumor volume better predicts prognosis than the conventional staging system based on tumor diameter in $\geq 45$-year-old patients with T1N0 PTC. Therefore, we suggest that future risk stratification systems should consider tumor volume as a prognostic factor for such patients.

\section{Conflicts of Interest}

Conflict of interest relevant to this article was not reported. 


\section{References}

1. Schlumberger MJ. Papillary and follicular thyroid carcinoma. N Engl J Med. 1998;338:297-306.

2. DeSantis CE, Lin CC, Mariotto AB, Siegel RL, Stein KD, Kramer JL, et al. Cancer treatment and survivorship statistics, 2014. CA Cancer J Clin. 2014;64:252-71.

3. Enewold L, Zhu K, Ron E, Marrogi AJ, Stojadinovic A, Peoples GE, et al. Rising thyroid cancer incidence in the United States by demographic and tumor characteristics, 1980-2005. Cancer Epidemiol Biomarkers Prev. 2009;18:784-91.

4. Lee JH, Lee ES, Kim YS, Won NH, Chae YS. BRAF mutation and AKAP9 expression in sporadic papillary thyroid carcinomas. Pathology. 2006;38:201-4.

5. Hall SF, Walker H, Siemens R, Schneeberg A. Increasing detection and increasing incidence in thyroid cancer. World J Surg. 2009;33:2567-71.

6. Davies L, Welch HG. Increasing incidence of thyroid cancer in the United States, 1973-2002. JAMA. 2006;295:2164-7.

7. Brito JP, Morris JC, Montori VM. Thyroid cancer: zealous imaging has increased detection and treatment of low risk tumours. BMJ. 2013;347:f4706.

8. Ahn HS, Kim HJ, Welch HG. Korea's thyroid-cancer "epidemic": screening and overdiagnosis. N Engl J Med. 2014;371: 1765-7.

9. Edge SB, Byrd DR, Compton CC, Fritz AG, Greene FL, Trotti A. AJCC cancer staging manual. 7th ed. New York: Springer; 2010.

10. Haugen BR, Alexander EK, Bible KC, Doherty GM, Mandel SJ, Nikiforov YE, et al. 2015 American Thyroid Association management guidelines for adult patients with thyroid nodules and differentiated thyroid cancer: The American Thyroid Association Guidelines Task Force on Thyroid Nodules and Differentiated Thyroid Cancer. Thyroid. 2016;26:1-133.
11. Tsai $\mathrm{CH}$, Lin $\mathrm{CM}$, Hsieh CC, Hsu WH, Wang HW, Wang LS. Tumor volume is a better prognostic factor than greatest tumor diameter in stage Ia non-small cell lung cancer. Thorac Cardiovasc Surg. 2006;54:537-43.

12. Davis KS, Lim CM, Clump DA, Heron DE, Ohr JP, Kim S, et al. Tumor volume as a predictor of survival in human papillomavirus-positive oropharyngeal cancer. Head Neck. 2016;38 Suppl 1:E1613-7.

13. Park KN, Kang KY, Hong HS, Jeong HS, Lee SW. Predictive value of estimated tumor volume measured by ultrasonography for occult central lymph node metastasis in papillary thyroid carcinoma. Ultrasound Med Biol. 2015;41:2849-54.

14. Anderson KL Jr, Youngwirth LM, Scheri RP, Stang MT, Roman SA, Sosa JA. T1a versus T1b differentiated thyroid cancers: do we need to make the distinction? Thyroid. 2016;26: 1046-52.

15. Wang LY, Nixon IJ, Palmer FL, Thomas D, Tuttle RM, Shaha $\mathrm{AR}$, et al. Comparable outcomes for patients with pT1a and pT1b differentiated thyroid cancer: Is there a need for change in the AJCC classification system? Surgery. 2014;156:1484-9.

16. Chereau N, Tresallet C, Noullet S, Godiris-Petit G, Tissier F, Leenhardt L, et al. Does the T1 subdivision correlate with the risk of recurrence of papillary thyroid cancer? Langenbecks Arch Surg. 2016;401:223-30.

17. Moon HJ, Yoon JH, Kwak JY, Chung WY, Nam KH, Jeong JJ, et al. Positive predictive value and interobserver variability of preoperative staging sonography for thyroid carcinoma. AJR Am J Roentgenol. 2011;197:W324-30.

18. Park JS, Son KR, Na DG, Kim E, Kim S. Performance of preoperative sonographic staging of papillary thyroid carcinoma based on the sixth edition of the AJCC/ UICC TNM classification system. AJR Am J Roentgenol. 2009;192:66-72. 